\title{
Newton's and Einstein's Gravity in a New Perspective for Planck Masses and Smaller Sized Objects
}

\author{
Espen Gaarder Haug \\ Norwegian University of Life Sciences, Ås, Norway \\ Email: espenhaug@mac.com
}

How to cite this paper: Haug, E.G. (2018) Newton's and Einstein's Gravity in a New Perspective for Planck Masses and Smaller Sized Objects. International Journal of Astronomy and Astrophysics, 8, 6-23. https://doi.org/10.4236/ijaa.2018.81002

Received: April 3, 2017

Accepted: February 6, 2018

Published: February 9, 2018

Copyright $\odot 2018$ by author and Scientific Research Publishing Inc. This work is licensed under the Creative Commons Attribution International License (CC BY 4.0).

http://creativecommons.org/licenses/by/4.0/

\section{(c) (i) Open Access}

\begin{abstract}
Here we derive Newton's and Einstein's gravitational results for any mass less than or equal to a Planck mass. All of the new formulas presented in this paper give the same numerical output as the traditional formulas. However, they have been rewritten in a way that gives a new perspective on the formulas when working with gravity at the level of the subatomic world. To rewrite the well-known formulas in this way could make it easier to understand the strengths and weaknesses in Newton's and Einstein's gravitation formulas at the subatomic scale, potentially opening them up for new important interpretations and extensions. For example, we suggest that the speed of gravity equal to that of light is actually embedded and hidden inside of Newton's gravitational formula.
\end{abstract}

\section{Keywords}

Gravitation, Gravitational Constant, Escape Velocity, Gravitational Time

Dilation, Schwarzschild Radius, Planck Length, Reduced Compton

Wavelength, Bending of Light, Red-Shift, Planck Mass

\section{Introduction}

In a recent paper, Haug [1] has rewritten many of Newton's and Einstein's gravitational results, without changing their output, into a quantized Planck form. However, his results only hold down to the scale of Planck mass sized objects. Here we derive similar results for any mass less than or equal to a Planck mass. Based on dimensional analysis [2], Newton's gravitational constant [3] can be written as a function of the reduced Planck constant, the Planck length, and the speed of light: 


$$
G_{p}=\frac{l_{p}^{2} c^{3}}{\hbar}
$$

Alternatively, this way of writing the gravitational constant can be obtained directly by solving the Planck length formula, [4] with respect to $G$. One could claim that this leads to a circular argument, since the Planck length is obtained from the gravitational constant. This circular problem is discussed and solved by Haug [2] [5], where he provides new theoretical insight that strongly indicates one can find $l_{p}$ independent of any knowledge of $G$. See also [6]. Further, based on the recent developments in mathematical atomism [7] [8], it is reasonable to think that the Planck length is among the most fundamental constants that could represent the diameter of an indivisible particle. We are not questioning whether or not big $G$ is a universal constant; we are asking if big $G$ could be a universal composite constant consisting of even more fundamental constants, and we have reason to think these are $c$, $\hbar$, and $l_{p}$.

There is still considerable uncertainty about the exact measurement of the gravitational constant. Experimentally, substantial progress has been made in recent years based on various methods. See, for example, [9] [10] [11] [12] [13]. Also, the relationship between physical constants from the microcosmos (subatomic world) and the macrocosmos (cosmos) plays an important role in physics. A continuous effort is being devoted to improving our measurements and understanding of these relationships. See, for example, [14].

The Planck form of the gravitational constant enables us to rewrite the Planck length as

$$
l_{p}=\sqrt{\frac{\hbar G_{p}}{c^{3}}}=\sqrt{\frac{\hbar \frac{l_{p}^{2} c^{3}}{\hbar}}{c^{3}}}
$$

and the mass of any subatomic particle with mass up or equal to the Planck mass, $m_{p}$, can be written as

$$
m=\frac{l_{p}}{\bar{\lambda}} m_{p}=\frac{l_{p}}{\bar{\lambda}} \sqrt{\frac{\hbar c}{G_{p}}}=\frac{l_{p}}{\bar{\lambda}} \sqrt{\frac{\hbar c}{\frac{l_{p}^{2} c^{3}}{\hbar}}}=\frac{\hbar}{\bar{\lambda}} \frac{1}{c}
$$

where $\bar{\lambda}$ is the reduced Compton wavelength of the mass in question. While the Planck mass as a function of $G$ was given by Planck in 1906, the relationship $m=\frac{l_{p}}{\bar{\lambda}} m_{p}$ was possibly first pointed out by Hoyle, Burbidge, and Narlikar in 1994, see [15]. In the special case of a Planck mass, we have $\bar{\lambda}=l_{p}$ (in equation 3) and we get

$$
m_{p}=\frac{\hbar}{l_{p}} \frac{1}{c}
$$

Using the gravitational constant in the Planck form, as well as the mass formula relationship above, we can easily rewrite a series of mathematical end results from Newton's and Einstein's gravitational formulas without changing 
their output values. The elements that will change are the input parameters and their mathematical form. Seeing well-known formulas in this new perspective seems to give new and additional insight that we believe can be useful.

\section{Newton's Universal Gravitational Force}

Newton's gravitational force is given by

$$
F_{G}=G_{p} \frac{m_{1} m_{2}}{R^{2}}
$$

Using the gravitational constant of the form $G_{p}=\frac{l_{p}^{2} c^{3}}{\hbar}$ and two subatomic particles with the same reduced Compton wavelength, we can rewrite Newton's gravitational force for two subatomic particles as

$$
\begin{gathered}
F=G_{p} \frac{m_{1} m_{2}}{R^{2}} \\
F=\frac{l_{p}^{2} c^{3}}{\hbar} \frac{\frac{\hbar}{\bar{\lambda}} \frac{1}{c} \frac{\hbar}{\bar{\lambda}} \frac{1}{c}}{R^{2}}=\frac{l_{p}^{2}}{\bar{\lambda}^{2}} \frac{\hbar c}{R^{2}}
\end{gathered}
$$

In the case where $R=\bar{\lambda}$, we get

$$
F=\frac{l_{p}^{2}}{\bar{\lambda}^{2}} \frac{\hbar c}{\bar{\lambda}^{2}}
$$

In the special case of two Planck masses, we have $\bar{\lambda}=l_{p}$ and we get the well-known Planck force

$$
F_{p}=\frac{\hbar c}{l_{p}^{2}}
$$

Naturally, it is questionable if Newtonian gravity holds at the Planck scale. The Planck mass is often assumed to play a role only at the extremely high energy scales where quantum gravity becomes important. No gravitational experiment has been done at anything close to Planck length or at Planck energies (Planck scale), so one should be careful about coming to any conclusions at this point in time. The main point here is to show the various Newtonian and also Einsteinian formulas in terms of what the author personally claims are the more fundamental constants, namely the Planck length, the Planck constant, and the speed of light, rather than what we believe is a composite constant, big $G$.

\section{The Speed of Gravity Hidden within Newton's Gravitational Formula?}

It is often assumed that the speed of gravity in Newton gravitational theory is instantaneous or alternatively that the Newton says nothing about how fast gravity should get transmitted. Here we will question this view and claim that the speed of gravity in the Newtonian formula must indeed be the speed of light, based on calibrating the formula when finding the gravitational constant. 
If Newton's gravitational constant actually is a composite constant as suggested, $G_{p}=\frac{l_{p}^{2} c^{3}}{\hbar}$, then interestingly the speed of gravity is actually embedded in Newton's gravitational theory, perhaps without Newton knowing about it. And as it is a composite, one would also have to know the Planck constant to back out the speed of light. Even if we have $c^{3}$ in the suggested composite version of the Newton gravitational constant, this always simply leads to $c$ in the gravitational force formula as shown in Equation (6).

We will claim that if the speed of gravity were different than $c$, then the empirically estimated gravitational constant has to be different than measured. Not only that, we will even claim that to some degree quantization in the Newton formula is also embedded in the observed gravitational constant. Had the Planck length even been different by $10 \%$, then this would change the gravitational constant by $20 \%$. Haug [5] has recently shown that the measurement error in the Planck length is exactly half of that of the gravitational constant. This is based on the assumption that the Planck length represents something fundamental that plays a central role in the quantum world and that gravity itself is ultimately affected by the Planck scale, even at the cosmic scale.

\section{Gravitational Acceleration Field}

The gravitational acceleration field in modern physics is given by

$$
g=\frac{G M}{r^{2}}
$$

This can be rewritten in quantized form for particles with a mass less than or equal to a Planck mass as

$$
\begin{gathered}
g=\frac{G_{p} m}{\bar{\lambda}^{2}} \\
g=\frac{\frac{l_{p}^{2} c^{3}}{\hbar} \frac{\hbar}{\bar{\lambda}} \frac{1}{c}}{\bar{\lambda}^{2}} \\
g=\frac{l_{p}^{2}}{\bar{\lambda}^{3}} c^{2}
\end{gathered}
$$

In the special case of a Planck mass, $\bar{\lambda}=l_{p}$ we get the well-known Planck mass acceleration $a_{p}=\frac{l_{p}^{2}}{l_{p}^{3}} c=\frac{c^{2}}{l_{p}}$; see [16] and [17]. An alternative is to try to define the maximum acceleration based on the Heisenberg uncertainty principle, see [18] [19]. If we have this maximum acceleration for one Planck second, we get to the speed of light:

$$
a_{p} t_{p}=\frac{c^{2}}{l_{p}} \frac{l_{p}}{c}=c
$$

This should be interpreted to mean that the Planck acceleration can only last for one Planck second, as nothing can move faster than the speed of light. This 
leads to a possibly interesting paradox. If the Planck-time is the shortest possible time something can undergo acceleration, then nothing with rest-mass can undergo Planck acceleration as this would mean it would reach the speed of light. And nothing with rest-mass can travel at the speed of light as this would lead to infinite kinetic energy, which was first pointed out by Einstein [20]. Haug [21] has recently suggested the Planck acceleration only can happen for a Planck mass particle and that this particle then only can last for one Planck second before dissolving into pure energy. This would at least be consistent with a mass accelerated to the speed of light, where the mass is no longer a mass but rather becomes energy. Could this extremely short lifespan of the Planck particle be the reason why we have not detected the Planck mass particle yet?

This may also mean that a Planck gravitational acceleration field can only last for one Planck second and that no rest-mass can survive it for more than a Planck second. The Planck acceleration field would give a speed of the object affected equal to the speed of light within one Planck second, something that is impossible for anything that has rest-mass. Our speculative interpretation is that only a Planck mass particle can be affected by the Planck acceleration field and that it will be dissolved into energy within one Planck second. This interpretation is at least consistent with something that can travel at the speed of light.

We can also find big $G$ from the gravitational acceleration parameter of any fundamental particle

$$
G=\frac{g}{m} \bar{\lambda}^{2}
$$

For example, we can find big $G$ from the gravitational acceleration field and the mass of a Planck mass particle

$$
G=\frac{g}{m} \bar{\lambda}^{2}=\frac{\frac{c^{2}}{l_{p}}}{\frac{\hbar}{l_{p}} \frac{1}{c}} l_{p}^{2}=\frac{l_{p}^{2} c^{3}}{\hbar}
$$

Or we can find big $G$ from the gravitational acceleration field and the mass of an electron

$$
G=\frac{g_{e}}{m_{e}} \bar{\lambda}_{e}^{2}=\frac{\frac{l_{p}^{2}}{\bar{\lambda}_{e}^{3}} c^{2}}{\frac{\hbar}{\bar{\lambda}_{e}} \frac{1}{c}} \bar{\lambda}_{e}^{2}=\frac{l_{p}^{2} c^{3}}{\hbar} \approx 6.67 \times 10^{-11}
$$

That is to say that $G$ is always the same no matter the particle; this even holds true for a half-Planck mass particle:

$$
G=\frac{g_{i}}{m_{i}} \bar{\lambda}_{i}^{2}=\frac{\frac{l_{p}^{2}}{\left(2 l_{p}\right)^{3}} c^{2}}{\frac{\hbar}{2 l_{p}} \frac{1}{c}}\left(2 l_{p}\right)^{2}=\frac{l_{p}^{2} c^{3}}{\hbar} \approx 6.67 \times 10^{-11}
$$


We can conclude that big $G$ is independent of the reduced Compton wavelength of the particle in question. With respect to this concept, we can call big $G$ a universal constant at the subatomic level as well, since it must be the same for any subatomic particle, at least inside our theoretical model, which is based on Newton.

\section{Gravitational Parameter}

The standard gravitational parameter is given by

$$
\mu=G m
$$

This can be rewritten in quantized form as

$$
\begin{gathered}
\mu_{p}=G_{p} m \\
\mu_{p}=G_{p} m \\
\mu_{p}=\frac{l_{p}^{2} c^{3}}{\hbar} N \frac{\hbar}{\bar{\lambda}} \frac{1}{c} \\
\mu_{p}=\frac{l_{p}^{2}}{\bar{\lambda}} c^{2}
\end{gathered}
$$

where $N$ is the number of particles with mass $m=\frac{\hbar}{\bar{\lambda}} \frac{1}{c}$ that make up the object in question.

\section{Escape Velocity}

The traditional Newtonian escape velocity [22] [23] is given by

$$
v_{e}=\sqrt{\frac{2 G m}{r}}
$$

where $G$ is the traditional gravitational constant, $m$ is the mass of the object we are "trying"to escape from, and $r$ is the radius we (for example a particle) are leaving from. Exactly the same escape velocity formula can be derived directly from Einstein's general relativity using the Schwarzschild metric, see [24].

Based on the gravitational constant written in the Planck form and the mass as written in the form given in the introduction, we can derive the escape velocity for a particle with mass equal or less than the Planck mass. We will assume the escape happens at a radius equal to the reduced Compton wavelength of the particle we are escaping from, $r=\bar{\lambda}$, this gives

$$
\begin{gathered}
v_{e}=\sqrt{\frac{2 G_{p} m}{\bar{\lambda}}} \\
v_{e}=\sqrt{\frac{2 \frac{l_{p}^{2} c^{3}}{\hbar} \frac{\hbar}{\bar{\lambda}} \frac{1}{c}}{\bar{\lambda}}} \\
v_{e}=\sqrt{\frac{2 \frac{l_{p}^{2}}{\bar{\lambda}} c^{2}}{\bar{\lambda}}}
\end{gathered}
$$




$$
v_{e}=\sqrt{2} \frac{l_{p}}{\bar{\lambda}} c
$$

This also means that the escape velocity of a particle is equal to the mass of the particle in question divided by the Planck mass and then multiplied by the speed of light and again multiplied by the square root of two

$$
v_{e}=\sqrt{2} \frac{l_{p}}{\bar{\lambda}} c=\sqrt{2} \frac{m}{m_{p}} c
$$

In the special case where the reduced Compton wavelength is $\sqrt{2} l_{p}$, we have an escape velocity of $c$, something that is well-known. This corresponds to a particle with mass

$$
m=\frac{\hbar}{\sqrt{2} l_{p}} \frac{1}{c}=\frac{1}{\sqrt{2}} m_{p}=\frac{1}{\sqrt{2}} \sqrt{\frac{\hbar c}{G}}
$$

This is sometimes called the Planck particle; it is the only particle where the Schwarzschild radius is equal to the reduced Compton wavelength.

Another interesting special case for the escape velocity is related to a particle with half the Planck mass in radius of $l_{p}$; this gives an escape velocity of $c$

$$
\begin{gathered}
v_{e}=\sqrt{\frac{2 G_{p} \frac{1}{2} m_{p}}{l_{p}}} \\
v_{e}=\sqrt{\frac{\frac{l_{p}^{2} c^{3}}{\hbar} \frac{\hbar}{l_{p}} \frac{1}{c}}{l_{p}}} \\
v_{e}=\sqrt{\frac{l_{p}^{2}}{l_{p}} c_{p}^{2}} \\
v_{e}=\frac{l_{p}}{l_{p}} c=c
\end{gathered}
$$

Motz and Epstein suggested in 1979 that there likely exists a very fundamental particle with half the Planck mass, [25]. However, they had no good explanation for why such a particle was so much heavier than any known observed particle. Haug [7] [8] has recently built a new fundamental theory around an indivisible particle with mass equal to half the Planck mass, where he has speculated on how such a particle could be the fundamental component of every observable mass. The Haug particle always moves at speed $C$ as measured with Einstein-Poincaré synchronized clocks and is quite different from what modern physics thinks of as particles. The indivisible particle is half a Planck mass when counter-striking (colliding) with another indivisible particle (so together a Planck mass), but only for a Planck second. When non-counter striking it is mass-less and the origin of energy. 
Haug [26] has recently suggested that an escape velocity below the Planck mass scale possibly should be linked to an escape probability. However, this is not clear and needs further investigation.

\section{Orbital Velocity}

The orbital velocity is given by

$$
v_{o} \approx \sqrt{\frac{G M}{r}}
$$

We can rewrite this in the form of the Planck gravitational constant and the Planck mass as

$$
\begin{gathered}
v_{o} \approx \sqrt{\frac{G_{p} m}{\bar{\lambda}}} \\
v_{o} \approx \sqrt{\frac{\frac{l_{p}^{2} c^{3}}{\hbar} \frac{\hbar}{\bar{\lambda}} \frac{1}{c}}{\bar{\lambda}}} \\
v_{o} \approx \frac{l_{p}}{\bar{\lambda}} c
\end{gathered}
$$

This can also be written as

$$
v_{o}=\frac{v_{e}}{\sqrt{2}}=\frac{l_{p}}{\lambda} C
$$

In the special case where $\bar{\lambda}=l_{p}$, then the orbital velocity is equal to $c$. The reduced Compton wavelength of a Planck mass is $l_{p}$. This also means the orbital velocity of a particle is equal to the mass of the particle in question divided by the Planck mass and then multiplied by the speed of light

$$
v_{o}=\frac{l_{p}}{\bar{\lambda}} c=\frac{m}{m_{p}} c
$$

\section{Gravitational Time Dilation at the Subatomic Level}

Einstein's gravitational time dilation [27] is given by

$$
t_{0}=t_{f} \sqrt{1-\frac{2 G m}{r c^{2}}}=t_{f} \sqrt{1-\frac{v_{e}^{2}}{c^{2}}}
$$

where $v_{e}$ is the traditional escape velocity. This means that the gravitational time dilation could have been derived from Newton's escape velocity, but to my knowledge Einstein is the first one to mention gravitational time dilation. We can rewrite this in the form of the quantized escape velocity (derived above).

$$
\begin{gathered}
t_{o}=t_{f} \sqrt{1-\frac{v_{e, p}^{2}}{c^{2}}} \\
t_{o}=t_{f} \sqrt{1-\frac{\left(\sqrt{2} \frac{l_{p}}{\lambda} c\right)^{2}}{c^{2}}}
\end{gathered}
$$




$$
t_{o}=t_{f} \sqrt{1-2 \frac{l_{p}^{2}}{\bar{\lambda}^{2}}}
$$

Notice that for a reduced Compton wavelength equal to the Planck length $\bar{\lambda}=l_{p}$, the formula above is invalid, or more precisely the time dilation becomes imaginary:

$$
t_{o}=t_{f} \sqrt{1-2 \frac{l_{p}^{2}}{l_{p}^{2}}}=t_{f} \sqrt{1-2}=t_{f} \sqrt{-1}=t_{f} i
$$

However, since $v_{e}$ for a Planck mass is $>c$, it is more precise to say that Einstein's time dilation formula possibly breaks down for a Planck mass particle (at the Planck scale).

Interestingly, for a half-Planck mass when at the Schwarzschild radius we get

$$
\begin{aligned}
& t_{o}=t_{f} \sqrt{1-\frac{v_{e}^{2}}{c^{2}}} \\
& t_{o}=t_{f} \sqrt{1-\frac{c^{2}}{c^{2}}} \\
& t_{o}=t_{f} \sqrt{1-1}=0
\end{aligned}
$$

In other words, only for half-Planck mass particles does time stand still. Haug has derived a new theory based on an indivisible particle with potential mass equal to half the Planck mass, and half the Planck rest-mass when colliding with another indivisible particle. Together these two indivisible particles are creating a Planck particle at the collision point. This particle likely only lasts for one Planck second before dissolving into energy again. Under atomism, time (and mass) are simply counter-strikes between indivisible particles. For a single indivisible particle, when not counter-striking, time also stands still; this is due to the concept that time under atomism is counter-strikes (clock ticks). Based on atomism, as well as Newton's corpuscular theory, light consists of indivisible particles traveling one after another. When they travel after each other they cannot counter-strike, since they all travel at the same speed and time stands still as "observed" from these particles.

\section{Circular orbits gravitational time dilation}

The time dilation for a clock at circular orbit ${ }^{1}$ is given by

$$
t_{0}=t_{f} \sqrt{1-\frac{3}{2} \frac{2 G m}{r c^{2}}}=\sqrt{1-\frac{3}{2} \frac{v_{e}^{2}}{c^{2}}}
$$

where $v_{e}$ is the traditional escape velocity. We can rewrite this in the form of the escape velocity for a subatomic particle, where we set the "radius" equal to the reduced Compton wavelength $r=\bar{\lambda}$,

$$
t_{o}=t_{f} \sqrt{1-\frac{3}{2} \frac{v_{e, p}^{2}}{c^{2}}}
$$

${ }^{1}$ At an orbital radius larger then $\frac{3}{2} r_{s}$ 


$$
\begin{aligned}
& t_{o}=t_{f} \sqrt{1-\frac{3}{2} \frac{\left(c \sqrt{2} \frac{l_{p}}{\bar{\lambda}}\right)^{2}}{c^{2}}} \\
& t_{o}=t_{f} \sqrt{1-3 \frac{l_{p}^{2}}{\bar{\lambda}^{2}}}
\end{aligned}
$$

\section{The Schwarzschild Radius}

The so-called Schwarzschild radius [28] [29] [30] [31] [32] of a mass $m$ is given by

$$
r_{s}=\frac{2 G m}{c^{2}}
$$

Rewritten as a function of the Planck units and the reduced Compton wavelength, it is given by

$$
\begin{gathered}
r_{s}=\frac{2 G_{p} m}{c^{2}} \\
r_{s}=\frac{2 \frac{l_{p}^{2} c^{3}}{\hbar} \frac{\hbar}{\bar{\lambda}} \frac{1}{c}}{c^{2}} \\
r_{s}=2 \frac{l_{p}^{2}}{\bar{\lambda}}
\end{gathered}
$$

We think this formula only make sense for sizes down to half-Planck masses and the Planck mass, but not for any mass smaller than a half-Planck mass. In 1979, Motz and Epstein [25] introduced a hypothetical particle with half of the Planck mass. Haug has derived an extensive theory around such a particle [7] [8]. For the half-Planck mass particle, we have a Schwarzschild radius of

$$
\begin{gathered}
r_{s}=\frac{2 G_{p} \frac{1}{2} m_{p}}{c^{2}} \\
r_{s}=\frac{G_{p} m_{p}}{c^{2}} \\
r_{s}=\frac{\frac{l_{p}^{2} c^{3}}{\hbar} \frac{\hbar}{l_{p}} \frac{1}{c}}{c^{2}} \\
r_{s}=l_{p}
\end{gathered}
$$

This indivisible particle has, according to Haug, a rest-mass equal to half the Planck mass and is likely the lowest mass a particle can take while the escape velocity still is $c$. It may be meaningless to talk about a Schwarzschild radius for fundamental particles with a mass less than half of the Planck mass. An electron, for example, does not have a valid Schwarzschild radius, even if the formula 33 above could be used to calculate a hypothetical Schwarzschild radius for an electron. The Schwarzschild radius for any "object" with a mass less than half of the Planck mass would be smaller than $l_{p}$. This is likely impossible and may be 
best understood from the recent renewal of atomism. The Schwarzschild radius of an indivisible particle is simply the diameter of the indivisible particle. Further, the Schwarzschild radius of a Planck mass is the length of two indivisible particles lying next to each other. Only for the indivisible particle is the Schwarzschild radius truly a radius in the sense that, at the depth of reality, it has to do with a perfectly spherical-shaped particle.

\section{Gravitational Light Deflection}

In 1884, Soldner derived work based on Newton's classical mechanics that predicted the following deflection of light

$$
\delta_{S}=\frac{2 G m}{c^{2} r}
$$

The angle of deflection in Einstein's general relativity theory [27] is twice that of the Soldner formula (1884)

$$
\delta_{G R}=\frac{4 G m}{c^{2} r}
$$

The solar eclipse experiment of Dyson, Eddington, and Davidson performed in 1919 confirmed [33] the idea that the deflection of light was very close to that predicted by Einstein's general relativity theory. That is 1.75 arcseconds compared to the 0.875 as predicted by Soldner's 1884 formula. ${ }^{2}$ This was one of the main reasons general relativity took off and partly replaced Newtonian gravitation.

Interestingly, Accioly and Ragusa [34] have shown that in semi-classical general relativity, the bending of light is dependent on $\frac{v}{C}$, and that when $v=0$, one gets the Soldner formula for Newtonian bending of light. When $v=c$ one gets the Einsteinian bending of light result. See also [35]. Sato and Sato [36] have recently pointed out that it looks like the two factor (double of Newton) in the light deflection likely are due to an unknown property of the photon rather than the bending of space. Hopefully, theoretical and experimental research will give us deeper insight into the deflection of light.

For a subatomic particle, Einstein's deflection of light can be rewritten as

$$
\begin{gathered}
\delta=\frac{4 G_{p} m}{c^{2} \bar{\lambda}} \\
\delta=\frac{4 \frac{l_{p}^{2} c^{3}}{\hbar} \frac{\hbar}{\bar{\lambda}} \frac{1}{c}}{c^{2} \bar{\lambda}} \\
\delta=4 \frac{l_{p}^{2}}{\bar{\lambda}^{2}}
\end{gathered}
$$

In the special case where we deal with a half-Planck mass $\bar{\lambda}=2 l_{p}$ we get

$$
\delta=4 \frac{l_{p}^{2}}{\left(2 l_{p}\right)^{2}}=1
$$

${ }^{2}$ In 1881, Soldner calculated the light deflection to be 0.84 arcseconds based on less accurate knowledge of the mass of the sun and speed of light than we have today. 
In terms of degrees, this is $1 \times \frac{648000}{\pi 3600} \approx 57.3^{\circ}$. Whether or not Einstein's light deflection formula truly makes sense at such short distances is an open question.

\section{Gravitational Redshift}

Einstein's gravitational redshift is given by

$$
\lim _{r \rightarrow+\infty} z(r)=\frac{1}{\sqrt{1-\frac{\frac{2 G m}{r}}{c^{2}}}}-1=\frac{1}{\sqrt{1-\frac{v_{e}^{2}}{c^{2}}}}-1
$$

where $r$ is the distance between the center of the mass of the gravitating body and the point at which the photon is emitted. For a subatomic particle, when we assume $r=\bar{\lambda}$, we can rewrite this as

$$
\begin{gathered}
\lim _{r \rightarrow+\infty} z(r)=\frac{1}{\sqrt{1-\frac{\sqrt{\frac{2 G m}{\bar{\lambda}}}}{c^{2}}}}-1 \\
\lim _{r \rightarrow+\infty} z(r)=\frac{1}{\sqrt{1-\frac{\frac{1}{l_{p}^{2} c^{3}} \frac{\hbar}{\bar{\lambda}} \frac{1}{c}}{c^{2}}}}-1 \\
\lim _{r \rightarrow+\infty} z(r)=\frac{1}{\sqrt{1-2 \frac{l_{p}^{2}}{\bar{\lambda}^{2}}}}-1
\end{gathered}
$$

By using a series expansion approximation of $\sqrt{1-2 \frac{l_{p}^{2}}{\bar{\lambda}^{2}}}$ when $\bar{\lambda} \gg l_{p}$ we get

$$
\sqrt{1-2 \frac{l_{p}^{2}}{\bar{\lambda}^{2}}} \approx 1-\frac{1}{2} 2 \frac{l_{p}^{2}}{\bar{\lambda}^{2}}
$$

and this gives

$$
\begin{gathered}
\lim _{r \rightarrow+\infty} z(r)=\frac{1}{1-\frac{1}{2} 2 \frac{l_{p}^{2}}{\bar{\lambda}^{2}}}-1 \\
\lim _{r \rightarrow+\infty} z(r)=\frac{1}{1-\frac{l_{p}^{2}}{\bar{\lambda}^{2}}}-\frac{1-\frac{l_{p}^{2}}{\bar{\lambda}^{2}}}{1-\frac{l_{p}^{2}}{\bar{\lambda}^{2}}} \\
\lim _{r \rightarrow+\infty} z(r)=\frac{\frac{l_{p}^{2}}{\bar{\lambda}^{2}}}{1-\frac{l_{p}^{2}}{\bar{\lambda}^{2}}} \approx \frac{l_{p}^{2}}{\bar{\lambda}^{2}}
\end{gathered}
$$

Several researchers have derived a similar redshift formula with no recourse to the general relativity theory, nor to the principle of equivalence, see [37] and [38] 


$$
\begin{gathered}
\lim _{r \rightarrow+\infty} z(r)=\frac{G_{p} m}{c^{2} \bar{\lambda}} \\
\lim _{r \rightarrow+\infty} z(r)=\frac{\frac{l_{p}^{2} c^{3}}{\hbar} \frac{\hbar}{\bar{\lambda}} \frac{1}{c}}{c^{2} \bar{\lambda}} \\
\lim _{r \rightarrow+\infty} z(r)=\frac{l_{p}^{2}}{\bar{\lambda}^{2}}
\end{gathered}
$$

Formula 43 is often considered to be an approximation to general relativity redshift, but one could also argue the other way around, that GR is an approximation to this formula. The prediction difference between the two formulas can only be observed in very high gravitational fields. One of the famous experiments that is claimed to have confirmed general relativity with very high precision is the Pound and Rebka [39] experiment. They measured the gravitational redshift in a tower over a distance of approximately 22.5 meters. This was an excellent experiment that got the same result as predicted by Einstein's general relativity theory. However, this experiment did not provide evidence that the general relativity theory is a complete theory. The experiment was done in a very weak gravitational field, where we know that the formula 43 should work just as well. However, the theory proved that Einstein was correct in the notion that gravity affects time, something that has been confirmed by a long series of experiments since then. To my knowledge, no one had assumed that gravity could affect time before Einstein.

\section{Table Summary}

Table 1 summarizes our rewritten versions of several gravitational formulas.

13. Any Deeper Meaning behind $\frac{l_{p}}{\bar{\lambda}}$ and $\frac{l_{p}^{2}}{\bar{\lambda}^{2}}$ ?

The fact that the factors $\frac{l_{p}}{\bar{\lambda}}$ and $\frac{l_{p}^{2}}{\bar{\lambda}^{2}}$ are showing up in several of the formulas in this paper, including the gravitational deflection of light, the redshift, and even Newton's gravitational force formula is particularly interesting. Haug [8] has recently shown that

$$
\frac{l_{p}}{\bar{\lambda}}=\sqrt{1-\frac{v_{\max }^{2}}{c^{2}}}
$$

where $v_{\max }=c \sqrt{1-\frac{l_{p}^{2}}{\bar{\lambda}^{2}}}$ is the maximum speed a uniform mass likely can take.

At this maximum speed, a fundamental mass (an electron, for example) will have reached a relativistic mass equal to the Planck mass. This is discussed in detail in the recent papers by Haug [2] [6] [8] [40]. If correct, this means that many of the $\frac{l_{p}}{\bar{\lambda}}$ and $\frac{l_{p}^{2}}{\bar{\lambda}^{2}}$ factors showing up in the quantified formulas could 
Table 1. Some of the standard gravitational relationships given by Newton and Einstein and their expression rewritten for Planck masses and beyond. The radius set for subatomic particles are equal to the reduced Compton wavelength of the particle in question.

\begin{tabular}{|c|c|c|c|}
\hline Units & Newton and Einstein form & "Wavelength"-form & Mass form \\
\hline $\begin{array}{l}\text { Gravitational } \\
\text { constant }\end{array}$ & $G \approx 6.67408 \times 10^{-11}$ & $G_{p}=\frac{l_{p}^{2} c^{3}}{\hbar}$ & \\
\hline $\begin{array}{l}\text { Newton's } \\
\text { gravitational force }\end{array}$ & $F=G \frac{m_{1} m_{2}}{r^{2}}$ & $F=\frac{\hbar c}{r^{2}} \frac{l_{p}^{2}}{\bar{\lambda}^{2}}$ & $F=\frac{\hbar c}{r^{2}} \frac{m^{2}}{m_{p}^{2}}$ \\
\hline Escape velocity & $v_{e}=\sqrt{\frac{2 G m}{r}}$ & $v_{e}=\frac{l}{\lambda}$ & $v_{e}=\sqrt{2} \frac{m}{m_{p}} c$ \\
\hline Orbital velocity & $v_{o}=\sqrt{\frac{G m}{r}}$ & & $v_{o}=\frac{m}{m_{p}} c$ \\
\hline $\begin{array}{l}\text { Gravitational } \\
\text { parameter }\end{array}$ & $\mu=G m$ & & $\mu=\frac{m}{m_{p}} l_{p} c^{2}$ \\
\hline $\begin{array}{c}\text { Gravitational } \\
\text { acceleration field }\end{array}$ & $g=\frac{G m}{r^{2}}$ & $g=\frac{l_{p}^{2}}{\bar{\lambda}^{3}} c^{2}$ & $g=\frac{m^{2}}{m_{p}^{2}} \frac{c^{2}}{\bar{\lambda}}$ \\
\hline $\begin{array}{l}\text { Gravitational time } \\
\text { dilation }\end{array}$ & $t_{0}=t_{f} \sqrt{1-\frac{2 G m}{r c^{2}}}=\sqrt{1-\frac{v_{e}^{2}}{c^{2}}}$ & $t_{o}=t_{f} \sqrt{1-2 \frac{l_{p}^{2}}{\bar{\lambda}^{2}}}$ & $t_{o}=t_{f} \sqrt{1-2 \frac{m^{2}}{m_{p}^{2}}}$ \\
\hline $\begin{array}{l}\text { Orbital time } \\
\text { dilation }\end{array}$ & $t_{0}=t_{f} \sqrt{1-3 \frac{G m}{r c^{2}}}=\sqrt{1-\frac{3}{2} \frac{v_{e}^{2}}{c^{2}}}$ & $t_{o}=t_{f} \sqrt{1-3 \frac{l_{p}^{2}}{\bar{\lambda}^{2}}}$ & $t_{o}=t_{f} \sqrt{1-3 \frac{m^{2}}{m_{p}^{2}}}$ \\
\hline Light deflection & $\delta=\frac{4 G m}{c^{2} r}$ & $\delta=4 \frac{l_{p}^{2}}{\bar{\lambda}^{2}}$ & $\delta=4 \frac{m^{2}}{m_{p}^{2}}$ \\
\hline $\begin{array}{l}\text { Schwarzschild } \\
\text { Radius }^{\mathrm{a}}\end{array}$ & $r_{s}=\frac{2 G m}{c^{2}}$ & $r_{s}=2 \frac{l_{p}^{2}}{\bar{\lambda}}$ & $r_{s}=2 l_{p} \frac{m}{m_{p}}$ \\
\hline $\begin{array}{l}\text { Gravitational } \\
\text { redshift GR }\end{array}$ & $\lim _{r \rightarrow+\infty} z(r)=\frac{1}{\sqrt{1-\frac{\frac{2 G m}{r}}{c^{2}}}}-1$ & $\lim _{r \rightarrow+\infty} z(r)=\frac{1}{\sqrt{1-2 \frac{l_{p}^{2}}{\bar{\lambda}^{2}}}}-1$ & $\frac{1}{\sqrt{1-2 \frac{m^{2}}{m_{p}^{2}}}}-1$ \\
\hline $\begin{array}{l}\text { Gravitational } \\
\text { redshift } A B S^{\mathrm{b}}\end{array}$ & $\lim _{r \rightarrow+\infty} z(r)=\frac{G m}{c^{2} r}$ & $\lim _{r \rightarrow+\infty} z(r)=\frac{l_{p}^{2}}{\bar{\lambda}^{2}}$ & $\frac{m^{2}}{m_{p}^{2}}$ \\
\hline
\end{tabular}

${ }^{a}$ We think this formula is only valid for particles with a larger or equal to half the Planck mass. ${ }^{\mathrm{b}}$ Based on the derivation by Adler, Bassin, and Shiffer; see [37] that is done totally independently of GR assumptions.

be quantum relativistic adjustments (that we not have been aware of until now), which are already there. Haug has shown that

$$
F=G \frac{m_{e} m_{e}}{r^{2}}=G \frac{m_{p} \sqrt{1-\frac{v_{\max }^{2}}{c^{2}}} m_{p} \sqrt{1-\frac{v_{\max }^{2}}{c^{2}}}}{r^{2}}=\frac{\hbar c}{r^{2}}\left(1-\frac{v_{\max }^{2}}{c^{2}}\right)=\frac{\hbar c}{r^{2}} \frac{l_{p}^{2}}{\bar{\lambda}^{2}}
$$

where $v_{\max }$ in this case is the speed the electron needs to take to reach a relativistic mass equal to the Planck mass, that is $v_{\max }=c \sqrt{1-\frac{l_{p}^{2}}{\bar{\lambda}_{e}^{2}}}$. The term $\frac{l_{p}^{2}}{\bar{\lambda}_{e}^{2}}$ is the well-known dimensionless gravitational coupling constant; see [41] [42] [43] [44], that is more commonly written in the form $\alpha_{G}=\frac{m_{e}^{2}}{m_{p}^{2}}$.

In an earlier paper [45], when looking at a possible connection between 
electromagnetism and gravity, Haug has also speculated that $\frac{l_{p}^{2}}{\bar{\lambda}^{2}}$ could a be conditional probability factor, related to the probability of gravity hits. The gravitational coupling constant could be both a quantum relativistic adjustment as well as a conditional probability factor at the same time; this is fully possible and further investigation should be encouraged.

The suggested maximum velocity seems to lead to the conclusion that Lorentz invariance breaks down for extremely high energy levels (at the Planck scale). That is to say for velocities and energies far above what currently achieved at the Large Hadron Collider. This is not the first gravitational theory modification or interpretation that would predict the breakage of Lorentz invariance at very high energy levels. Quantum gravity theories in general predict breaks in Lorentz invariance at the Planck scale, see for example [46]. We are not in any way claiming that we have a presented a new ("complete") quantum gravity theory here. Rather we are suggesting that a new interpretation of what is already being examined could be embedded in the Newtonian and Einsteinian formulas, which may also be pointing towards concepts similar to those being explored in some quantum gravity theories. Further investigation is naturally needed on this front.

\section{Conclusions}

By making the gravitational constant a function form of the reduced Planck constant, one can easily rewrite many of the end results from Newton's and Einstein's gravitation in quantized form. This has been done recently for masses above or equal to the Planck mass. In this paper, this framework has been extended to hold below Planck mass sized objects as well. This gives the same numerical end results as those obtained by Newton and Einstein, but now provides new insight about the subatomic world, or at least insight in how well or not so well Newton's and Einstein's gravitational theories fit the subatomic world. How these new formulas, which only rely on the Planck length, the Planck constant, the speed of light, and the reduced Compton wavelength, should be interpreted is open to discussion.

However, we have suggested that the speed of gravity equal to the speed of light actually is embedded in the Newton formula, and that even quantization related to the Planck length and the Planck scale is embedded in the Newton gravitational formula.

\section{References}

[1] Haug, E.G. (2016) Planck Quantization of Newton and Einstein Gravitation. International Journal of Astronomy and Astrophysics, 6, 206-217. https://doi.org/10.4236/ijaa.2016.62017

[2] Haug, E.G. (2016) The Gravitational Constant and the Planck Units: A Simplification of the Quantum Realm. Physics Essays, 29, 4 p.

[3] Newton, I. (1686) Philosophiae Naturalis Principia Mathematica. Joseph Streater, London. 
[4] Planck, M. (1906) The Theory of Radiation. Dover 1959 Translation, (1914 version) P. Blakiston's Sons \& Co., Philadelphia, PA.

[5] Haug, E.G. (2017) Can the Planck Length Be Found Independent of Big G? Applied Physics Research, 9, 7 p.

[6] Haug, E.G. (2016) A New Solution to Einstein's Relativistic Mass Challenge Based on Maximum Frequency. http://vixra.org/abs/1609.0083

[7] Haug, E.G. (2014) Unified Revolution: New Fundamental Physics. E.G.H. Publishing, Oslo.

[8] Haug, E.G. (2016) The Planck Mass Particle Finally Discovered! Good Bye to the Point Particle Hypothesis! http://vixra.org/pdf/1607.0496v6.pdf

[9] Bisnovatyi-Kogan, G.S. (2006) Checking the Variability of the Gravitational Constant with Binary Pulsars. International Journal of Modern Physics D, 15, 1047. https://doi.org/10.1142/S0218271806008747

[10] Fixler, B., Foster, G.T., McGuirk, J.M. and Kasevich, M.A. (2007) Atom Interferometer Measurement of the Newtonian Constant of Gravity. Science, 315, 74-77. https://doi.org/10.1126/science.1135459

[11] Galli, S., Melchiorri, A., Smoot, G.F. and Zahn, O. (2009) From Cavendish to Planck: Constraining Newton's Gravitational Constant with CMB Temperature and Polarization Anisotropy. Physical Review D, 80, 023508-023514. https://doi.org/10.1103/PhysRevD.80.023508

[12] Rosi, G., Sorrentino, F., Cacciapuoti, L., Prevedelli, M. and Tino, G.M. (2014) Precision Measurement of the Newtonian Gravitational Constant Using Cold Atoms. Nature, 510, 518-521. https://doi.org/10.1038/nature13433

[13] Schlamminger, S. (2014) Fundamental Constant: A Cool Way to Measure Big G. Nature, 510, 478-480. https://doi.org/10.1038/nature13507

[14] Collaboration, P., et al. (2015) Planck Intermediate Results-XXIV: Constraints on Variations in Fundamental Constants. Astronomy \& Astrophysics, 580, A22.

[15] Hoyle, F., Burbidge, G. and Narlikar, J. (1994) The Basic Theory Underlying the Quasi-Steady State Cosmology. Proceedings of the Royal Society A, 448, 191-212. https://doi.org/10.1098/rspa.1995.0012

[16] Scarpetta, G. (1984) Relativistic Kinematics with Caianiello's Maximal Proper Acceleration. Lettere al Nuovo Cimento, 41, 51-58. https://doi.org/10.1007/BF02748462

[17] Haug, E.G. (2016) Charged Particle Radiation Power at the Planck Scale. http://vixra.org/pdf/1604.0228v2.pdf

[18] Caianiello, E.E. (1984) Maximal Acceleration as a Consequence of Heisenberg's Uncertainty Relations. Lettere al Nuovo Cimento, 41, 370-372. https://doi.org/10.1007/BF02748378

[19] Papini, G. (2002) Revisiting Caianiello's Maximal Acceleration. IL Nuovo Cimento $B, 117,1325$.

[20] Einstein, A. (1916) Relativity: The Special and the General Theory. Lawson, R., Trans. Crown Publishers, New York.

[21] Haug, E.G. (2017) The Incompatibility of the Planck Acceleration and Modern Physics? http://vixra.org/abs/1710.0340

[22] Schutz, B. (2003) Gravity from the Ground Up. Cambridge University Press, Cambridge. https://doi.org/10.1017/CBO9780511807800

[23] Tipler, P.A. and Llewellyn, R.A. (2012) Modern Physics. 6th Edition, W. H. Freeman and Company, New York. 
[24] Augousti, A.T. and Radosz, A. (2006) An Observation on the Congruence of the Escape Velocity in Classical Mechanics and General Relativity in a Schwarzschild Metric. European Journal of Physics, 376, 331-335. https://doi.org/10.1088/0143-0807/27/2/015

[25] Motz, L. and Epstein, J. (1979) The Gravitational Charge $1 / 2 \sqrt{\hbar c}$ as a Unifying Principle in Physics. IL Nuovo Cimento, 51, 88-113.

[26] Haug, E.G. (2016) Escape Velocity at the Subatomic Level Leads to Escape Probability. http://vixra.org/pdf/1611.0053v1.pdf

[27] Einstein, A. (1916) Näherungsweise integration der feldgleichungen der gravitation. Sitzungsberichte der Königlich Preussischen Akademie der Wissenschaften Berlin.

[28] Schwarzschild, K. (1916) Über das gravitationsfeld einer kugel aus inkompressibler flussigkeit nach der einsteinschen theorie. Sitzungsberichte der Deutschen Akademie der Wissenschaften zu Berlin, Klasse fur Mathematik, Physik, und Technik, 424.

[29] Schwarzschild, K. (1916) Über das gravitationsfeld eines massenpunktes nach der einsteinschen theorie. Sitzungsberichte der Deutschen Akademie der Wissenschaften zu Berlin, Klasse fur Mathematik, Physik, und Technik, 189.

[30] Hilbert, D. (1917) Nachr. ges. wiss. Göttingen, Math. Phys.

[31] Antoci, S. David Hilbert and the Origin of the "Schwarzschild Solution". https://arxiv.org/abs/physics/0310104

[32] Crothers, S.J. (2006) A Brief History of Black Holes. Progress in Physics, 2, 54-57. http://www.ptep-online.com/2006/PP-05-10.PDF

[33] Dyson, F., Eddington, A. and Davidson, C. (1920) A Determination of the Deflection of Light by the Sun's Gravitational Field, from Observations Made at the Total Eclipse of May 29, 1919. Philosophical Translation Royal Society, 220, 291-333.

[34] Accioly, A. and Ragusa, S. (2002) Gravitational Deflection of Massive Particles in Classical and Semiclassical General Relativity. Classical and Quantum Gravity, 19, 5429. https://doi.org/10.1088/0264-9381/19/21/308

[35] Patla, B., Nemiroff, R., Hoffmann, D. and Zioutas, K. (2014) Flux Enhancement of Slow-Moving Particles by Sun or Jupiter: Can They Be Detected on Earth? The Astrophysical Journal, 780, 158. http://iopscience.iop.org/article/10.1088/0004-637X/780/2/158/pdf

[36] Sato, M. and Sato, H. (2016) Light Bending by Gravity: Back to Space and Time from Spacetime. Physics Essays, 29, 423-426.

[37] Adler, R., Bazin, M. and Schiffer, M. (1965) Introduction to General Relativity. McGraw-Hill, New York.

[38] Evans, R. and Dunning-Davies, J. (2004) The Gravitational Red-Shift. https://arxiv.org/abs/gr-qc/0403082

[39] Pound, R.V. and Rebka Jr., G.A. (1959) Gravitational Red-Shift in Nuclear Resonance. Physical Review Letters, 3, 439-441. https://doi.org/10.1103/PhysRevLett.3.439

[40] Haug, E.G. (2017) The Ultimate Limits of the Relativistic Rocket Equation: The Planck Photon Rocket. Acta Astronautica, 136, 144-147. https://doi.org/10.1016/j.actaastro.2017.03.011

[41] Silk, J. (1977) Cosmogony and the Magnitude of the Dimensionless Gravitational Coupling Constant. Nature, 265, 710-711. https://doi.org/10.1038/265710a0

[42] Rozental, I.L. (1980) On the Numerical Values of the Fine-Structure Constant and the Gravitational Constant. Soviet Journal of Experimental and Theoretical Physics Letters, 31, 19-27. 
[43] de Oliveira Neto, M. (2005) Using the Dimensionless Newton Gravity Constant $\bar{\alpha}_{g}$ to Estimate Planetary Orbits. Chaos, Solitons and Fractals, 24, 19-27. https://doi.org/10.1016/j.chaos.2004.07.032

[44] Burrows, A.S. and Ostriker, J.P. (2013) Astronomical Reach of Fundamental Physics. Proceedings of the National Academy of Sciences of the United States of America, 111, 31-36.

[45] Haug, E.G. (2016) Unification of Gravity and Electromagnetism: Gravity Electromagnetism: A Probability Interpretation of Gravity.

http://www.vixra.org/pdf/1604.0208v4.pdf

[46] Kislat, F., Krawczynski, H. (2017) Planck-Scale Constraints on Anisotropic Lorentz and CPT Invariance Violations from Optical Polarization Measurements. Physical Review D, 95, 083013. https://doi.org/10.1103/PhysRevD.95.083013 